\title{
Synthesis and characterization of silicon nanorod on n-type porous silicon
}

\begin{abstract}
This work reports a new method for growing semiconductor nanorods on a porous silicon substrate. After preparation of n-type porous silicon samples, a thin layer of gold was deposited on them. Gold deposited samples were annealed at different temperatures. The structural, thermal, and optical properties of the samples were studied using a field emission scanning electron microscope (FESEM), photoacoustic spectroscopy, and photoluminescence spectroscopy, respectively. FESEM analysis revealed that silicon nanorods of different sizes grew on the annealed samples. Thermal behavior of the samples was studied using photoacoustic spectroscopy. Photoluminescence spectroscopy showed that the emission peaks were degraded by gold deposition and attenuated for all samples by annealing.
\end{abstract}

Keyword: Silicon nanorod; Porous silicon; Thermal behavior; Gold 